\title{
Many-body Majorana-like zero modes without gauge symmetry breaking
}

\author{
V. Vadimov $\odot,{ }^{1,2,3}$ T. Hyart, ${ }^{4,5}$ J. L. Lado $\odot,{ }^{4}$ M. Möttönen $\odot,{ }^{1,6}$ and T. Ala-Nissila $\odot^{2,7}$ \\ ${ }^{1}$ QCD Labs, QTF Centre of Excellence, Department of Applied Physics, Aalto University, P.O. Box 13500, FI-00076 Aalto, Espoo, Finland \\ ${ }^{2}$ MSP Group, QTF Centre of Excellence, Department of Applied Physics, Aalto University, P.O. Box 11000, FI-00076 Aalto, Espoo, Finland \\ ${ }^{3}$ Institute for Physics of Microstructures, Russian Academy of Sciences, 603950 Nizhny Novgorod, GSP-105, Russia \\ ${ }^{4}$ Department of Applied Physics, Aalto University, FI-00076 Aalto, Espoo, Finland \\ ${ }^{5}$ International Research Centre MagTop, Institute of Physics, Polish Academy of Sciences, Al. Lotników 32/46, 02-668 Warsaw, Poland \\ ${ }^{6}$ VTT Technical Research Centre of Finland Ltd., QTF Center of Excellence, P.O. Box 1000, FI-02044 VTT, Finland \\ ${ }^{7}$ Interdisciplinary Centre for Mathematical Modelling, Department of Mathematical Sciences, Loughborough University, \\ Loughborough LE11 3TU, United Kingdom
}

(Received 18 November 2020; revised 5 March 2021; accepted 8 March 2021; published 1 April 2021)

\begin{abstract}
Topological superconductors represent one of the key hosts of Majorana-based topological quantum computing. Typical scenarios for one-dimensional (1D) topological superconductivity assume a broken gauge symmetry associated to a superconducting state. However, no interacting 1D many-body system is known to spontaneously break gauge symmetries. Here, we show that zero modes emerge in a many-body system without gauge symmetry breaking and in the absence of superconducting order. In particular, we demonstrate that Majorana zero modes of the symmetry-broken superconducting state are continuously connected to these zero-mode excitations, demonstrating that zero-bias anomalies may emerge in the absence of gauge symmetry breaking. We demonstrate that these many-body zero modes share the robustness features of the Majorana zero modes of symmetry-broken topological superconductors. We further show that the interface between the interacting model and a 1D topological superconductor does not support Majorana modes. We introduce a bosonization formalism to analyze these excitations and show that a ground state analogous to a topological superconducting state can be analytically found in a certain limit. Our results demonstrate that robust Majorana-like zero modes may appear in a many-body system without gauge symmetry breaking, thus introducing a family of protected excitations with no single-particle analogs.
\end{abstract}

DOI: 10.1103/PhysRevResearch.3.023002

\section{INTRODUCTION}

Superconductivity in topological quantum materials has become one of the most fertile topics in modern condensed matter physics [1,2]. The search for topological superconductors has been motivated by the emergence of topological excitations, known as Majorana zero modes [3], and by their potential for topological quantum computing [4-13]. A variety of solid-state materials have been explored in recent years with the goal of engineering Majorana bound states, including superconducting nanowires [14-22], atomically engineered chains [23-27], topological insulators [28-32], phase-controlled Josephson junctions [33,34], helical quantum Hall edge states of graphene [35] with controllable magnetic $[36,37]$ and superconducting gaps, antiferromagnetic topological superconductors [38-40], and van der Waals heterostructures [41]. These different platforms rely on the engineering of a specific kind of an effective $p$-wave superconducting state, the nontrivial topological prop-

Published by the American Physical Society under the terms of the Creative Commons Attribution 4.0 International license. Further distribution of this work must maintain attribution to the author(s) and the published article's title, journal citation, and DOI. erties of which give rise to the emergence of Majorana zero modes [42].

Majorana bound states and unconventional superconductors in general rely on a single-particle description of the effective excitations. In particular, conventional proposals for Majorana bound states in topological superconductors rely on explicitly broken gauge symmetry which is associated with the existence of a nonzero superfluid order parameter [42].

In this scenario, the existence of Majorana bound states in the presence of particle-particle interactions has been established in terms of a renormalization of the single-particle mean-field parameters [43-47]. Although additional subtle many-body effects are prone to appear in this regime [43,48-50], the Majorana zero remains to exist.

However, the scenario for systems lacking symmetry breaking is distinctively different [51-60]. Namely, particleparticle interactions cannot be reinterpreted as a renormalization of single-particle terms as in a symmetry-broken state. For typical single-particle models of topological superconductivity, the pairing term explicitly breaks the gauge symmetry since the term is not $\mathrm{U}(1)$ gauge symmetric. A finite pairing term is a hallmark of superconductivity, and thus such symmetry breaking is natural for proposals that involve three-dimensional superconductors [61]. However, for a purely one-dimensional (1D) system, the situation is dramatically different, as spontaneous symmetry breaking with finite pairing does not take place $[62,63]$. In particular, 
interacting 1D models have a ground state that is gauge symmetric with a vanishing expectation value of superconducting pairing [62,63], and consequently their effective single-particle Hamiltonian does not host Majorana zero modes [42,61]. Thus, whether or not Majorana zero modes may appear in the absence of gauge symmetry breaking is a major outstanding question.

In this work, we demonstrate that robust zero modes appear in a $1 \mathrm{D}$ many-body model without gauge symmetry breaking. The model we focus on would give rise to a topological superconductor at the mean-field level if the gauge symmetry were explicitly broken. We demonstrate that no such gauge symmetry breaking is required for the emergence of Majorana-like zero modes, establishing a peculiar paradigm of quantum many-body excitations with no single-particle analog. Despite their fundamental differences to Majorana zero modes, we demonstrate that these two types of many-body excitations share many properties, including robustness to perturbations and disorder.

Our paper is organized as follows. In Sec. II, we introduce the many-body model, highlighting the emergence of zeromode resonances. In Sec. III, we demonstrate the robustness of the zero modes to a variety of perturbations. In Sec. IV, we show the connection between these resonant zero modes and Majorana bound states. In Sec. V, we demonstrate the emergence of the edge modes from a continuum bosonization formalism. Section VI summarizes our results and Appendices A-C discuss some technical details such as critical points, Green's functions, and persistent current in a ring.

\section{ZERO MODES IN QUANTUM MANY-BODY CHAINS}

We study a $1 \mathrm{D}$ chain of $L$ spinless fermions with interactions between the neighboring sites as illustrated in Fig. 1(a). The system is described by the following Hamiltonian:

$$
\begin{aligned}
\hat{H}_{I}= & -t \sum_{j=1}^{L-1}\left(\hat{c}_{j+1}^{\dagger} \hat{c}_{j}+\hat{c}_{j}^{\dagger} \hat{c}_{j+1}\right)-\mu \sum_{j=1}^{L} \hat{c}_{j}^{\dagger} \hat{c}_{j} \\
& -V \sum_{j=1}^{L-1}\left(\hat{c}_{j+1}^{\dagger} \hat{c}_{j+1}-\frac{1}{2}\right)\left(\hat{c}_{j}^{\dagger} \hat{c}_{j}-\frac{1}{2}\right),
\end{aligned}
$$

where $t$ is the strength of the particle hopping between neighboring sites, $\hat{c}_{j}^{\dagger}$ and $\hat{c}_{j}$ are the fermionic creation and annihilation operators at site $j$, respectively, $\mu$ is the chemical potential, and $V$ is the strength of the interactions between the fermions. Such a system can be mapped onto a spin- $\frac{1}{2}$ anisotropic XXZ chain in a longitudinal field using the JordanWigner transformation, resulting in

$$
\hat{H}_{I}=\sum_{j=1}^{L-1}\left[2 t\left(\hat{s}_{j}^{x} \hat{s}_{j+1}^{x}+\hat{s}_{j}^{y} \hat{s}_{j+1}^{y}\right)-V \hat{s}_{j}^{z} \hat{s}_{j+1}^{z}\right]-\mu \sum_{j=1}^{L} \hat{s}_{j}^{z},
$$

where $\left\{\hat{s}_{j}^{\alpha}\right\}_{\alpha=x, y, z}$ denote the spin- $\frac{1}{2}$ operators for site $j$. This model is integrable by the means of the Bethe ansatz $[64,65]$. The resulting phase diagram at zero temperature and otherwise in the thermodynamic limit is shown in Fig. 1(b) [66]. We focus on the region of the diagram corresponding to the attractive interactions between the fermions $V>0$, where two different phases exist. Phase separation takes place at (a)

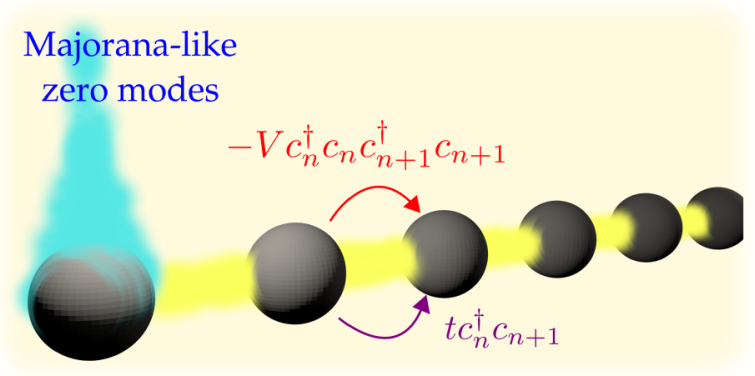

(b)

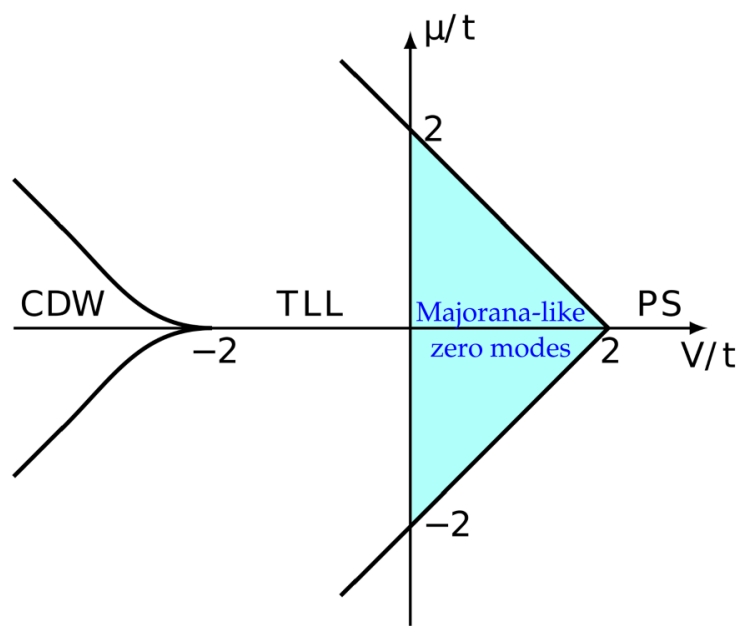

FIG. 1. (a) Schematic illustration of the interacting onedimensional model we study. The Majorana-like zero-energy mode at the left edge of the chain is highlighted with cyan color. The fermionic creation and annihilation operators at site $n$ (gray sphere) are denoted by $\hat{c}_{n}^{\dagger}$ and $\hat{c}_{n}$, respectively. The strength of the particle hopping between neighboring sites (yellow color) is given by $t$, and $V$ describes the strength of the interactions between the fermions. (b) The phase diagram of the Hamiltonian (1) at zero temperature. Here, $\mu$ denotes the chemical potential. Regions corresponding to a phase separation (PS), Tomonaga-Luttinger liquid (TLL), and charge density wave (CDW) appear in addition to Majorana-like zero modes (cyan color).

$|\mu|>2 t-V$ where the ground state corresponds, depending on the sign of $\mu$, to the vacuum state or to the completely filled band. In this phase, zero-energy modes which mix the number of particles are known to exist [67]. The other phase corresponds to the Tomonaga-Luttinger liquid [68-70] which is our main focus here. Remarkably, we have been able to explicitly construct the ground state at the critical point $V=2 t$, $\mu=0$ as shown in Appendix A. At this point it appears to be $L+1$ times degenerate, where the different degenerate states correspond to the different numbers of particles.

Treated within the mean-field approximation such a model gives rise to the well-known Kitaev model described by the Hamiltonian

$$
\begin{aligned}
\hat{H}_{K}= & -t \sum_{j=1}^{L-1}\left(\hat{c}_{j+1}^{\dagger} \hat{c}_{j}+\hat{c}_{j}^{\dagger} \hat{c}_{j+1}\right)-\mu \sum_{j=1}^{L} \hat{c}_{j}^{\dagger} \hat{c}_{j} \\
& +\sum_{j=1}^{L-1}\left(\Delta_{j} \hat{c}_{j+1}^{\dagger} \hat{c}_{j}^{\dagger}+\Delta_{j}^{*} \hat{c}_{j} \hat{c}_{j+1}\right),
\end{aligned}
$$


where the superconducting order parameter $\Delta_{j}$ is determined self-consistently as $\Delta_{j}=V\left\langle\hat{c}_{j} \hat{c}_{j+1}\right\rangle$. The Kitaev model spontaneously breaks the gauge symmetry which is present in the original model (1). For $L \gg t /|\Delta|$ this model hosts Majorana zero modes localized at the ends of the chain [42] whereas the other excited states are separated from the ground state by $|\Delta|$ in the bulk of the chain.

\section{A. Local density of states}

The local density of states, or the spectral function, of the chain is defined as

$$
\begin{aligned}
A(j, \omega)= & \left\langle\Psi_{0}\right| \hat{c}_{j} \delta\left(\hbar \omega-\hat{H}_{I}+E_{0}\right) \hat{c}_{j}^{\dagger} \\
& +\hat{c}_{j}^{\dagger} \delta\left(\hbar \omega+\hat{H}_{I}-E_{0}\right) \hat{c}_{j}\left|\Psi_{0}\right\rangle \\
= & \sum_{m}\left[\left|\left\langle\Psi_{0}\left|\hat{c}_{j}\right| \Psi_{m}\right\rangle\right|^{2} \delta\left(\hbar \omega-E_{m}+E_{0}\right)\right. \\
& \left.+\left|\left\langle\Psi_{0}\left|\hat{c}_{j}^{\dagger}\right| \Psi_{m}\right\rangle\right|^{2} \delta\left(\hbar \omega-E_{0}+E_{m}\right)\right],
\end{aligned}
$$

where $\left|\Psi_{0}\right\rangle$ is the ground state with energy $E_{0}$ and $\left|\Psi_{m}\right\rangle$ are all the eigenstates of the system corresponding to energies $E_{m}$. The spectral function can be evaluated using exact diagonalization of the Hamiltonian for the short chains or using a kernel polynomial method with matrix product states (KPMMPS) [71-77] for the reasonably long chains.

The results of our calculations are shown in Fig. 2. We observe clear zero-bias peaks at the edges of the chain, corresponding to Majorana-like zero modes. We find that these zero-energy peaks appear for all considered chemical potentials $\mu$. Similarly to Majorana edge modes, the peaks split for short chains as shown in Fig. 2(b), stemming from the hybridization of the excitations at the opposite edges. With increasing chain length $L$, the edge peaks move towards zero energy, constituting a zero-energy resonance in the limit $L \rightarrow$ $\infty$. Next, we systematically examine how the splitting of these edge modes depends on the system size. Interestingly, their behavior is different from that of Majorana zero modes in topological superconductors.

\section{B. Peak scaling}

The nature of the above-found edge modes can be studied by inspecting the scaling properties of the peak splitting. From the series expression in Eq. (3), we observe that the peaks are located at energies $\pm\left[E_{0}\left(N_{0} \pm 1\right)-E_{0}\right]$, where $E_{0}(N)$ is the energy of the ground state in the subspace with $N$ particles and $N_{0}$ is the number of particles in the global ground state of the system. Thus, the peak splitting $\hbar \omega_{\mathrm{p}}$ appears to be equal to $\left[E_{0}\left(N_{0}+1\right)+E_{0}\left(N_{0}-1\right)\right] / 2-E_{0}$ and its dependence of the chain length $L$ is shown in Fig. 3(a). For comparison, Fig. 3(a) also shows the splitting for a noninteracting chain, which corresponds to the level spacing at the Fermi energy. We observe that the splitting scales as $C(V) / L$, in stark contrast to the mean-field case where the splitting of the Majorana peaks decays exponentially with an increasing length of the chain.

In the case of conventional Majorana states, the exponential dependence arises because of two reasons. First, the bulk of the system has a gap stemming from the finite pairing. Since the Majorana zero modes are located inside the gap in the bulk of the system, they need to decay exponentially,
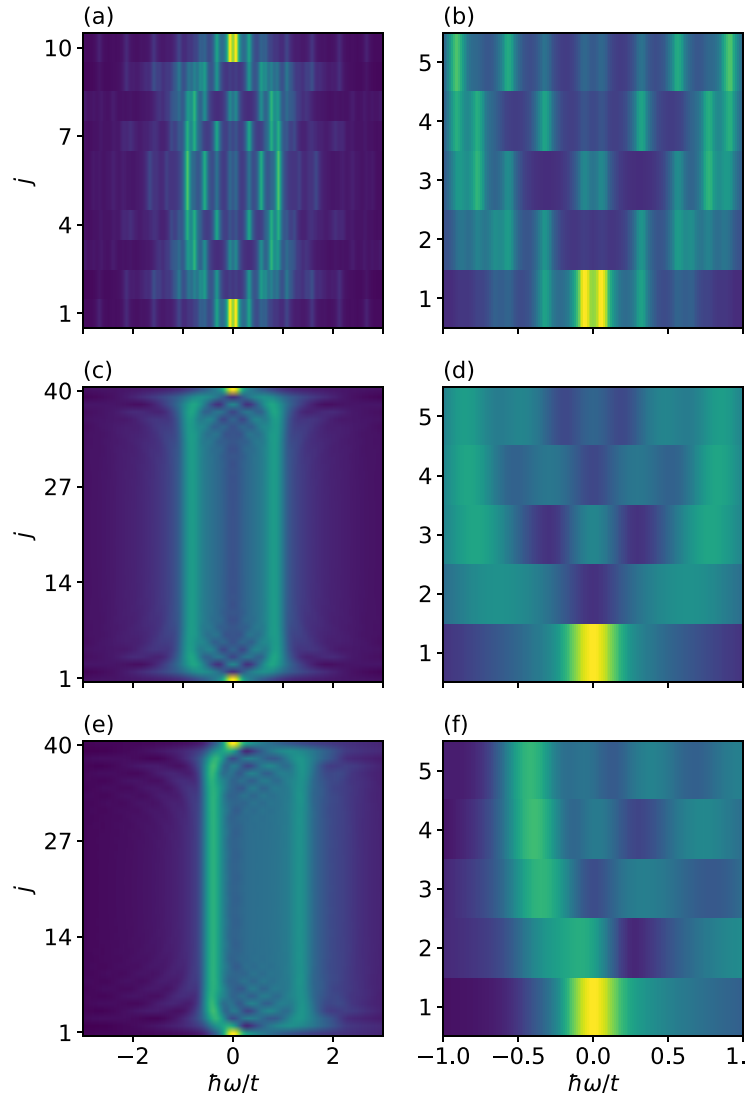

(d)
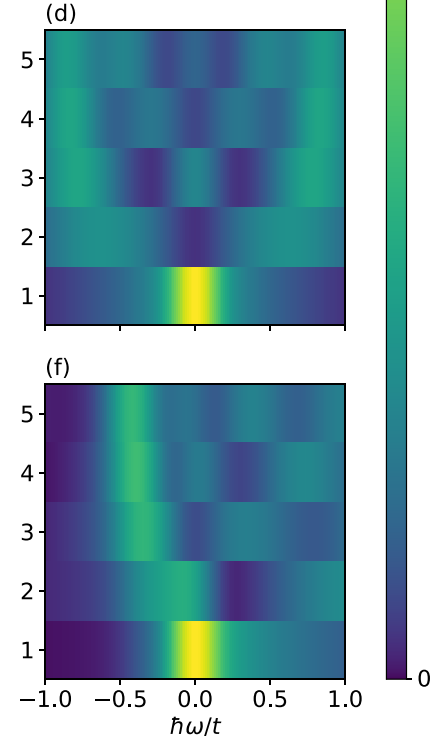

FIG. 2. Local density of states $A(j, \omega)$ as a function of the energy $\omega$ and the site index of the chain $j$ for (a), (b) $L=10, \mu=0$, (c), (d) $L=40, \mu=0$, (e), (f) $L=40, \mu=-0.2 \times t$. The interaction strength is $V=1.5 \times t$ in all panels. Note that (b), (d), and (f) simply show the the local density of states in the vicinity of the edge of the chain. The data in (a) and (b) have been obtained using exact diagonalization of the Hamiltonian (1) and the other data are obtained using the KPM-MPS method.

which gives rise to a hybridization between the zero modes that decays exponentially with the system size. Second, the induced superconductivity may be considered to arise from coupling to an infinite superconductor. Since the Majorana wire can exchange Cooper pairs with the infinite superconductor,
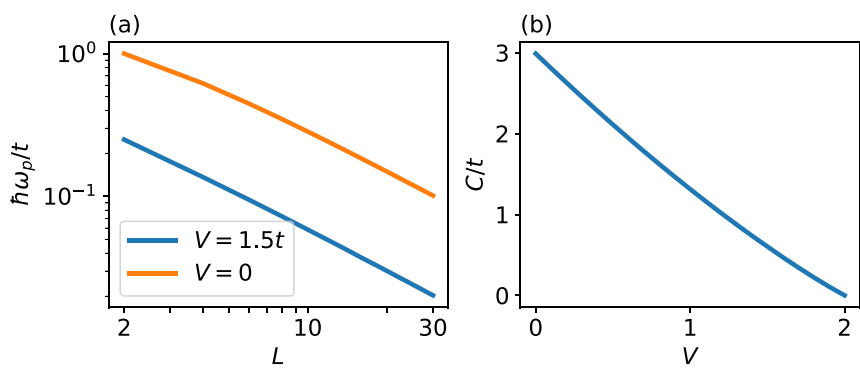

FIG. 3. (a) Energy splitting of the edge-mode peaks in the local density of states as a function of chain length $L$ for a chemical potential $\mu=0$ and interaction strength $V$ as indicated. (b) The scaling coefficient $C$, defined as the edge-mode energy splitting multiplied by $L$, as a function of the interaction strength $V$. All data are obtained using the KPM-MPS method. 
there is no energy cost for adding particles to the system. As a result of these two effects, the states with $N_{0}$ and $N_{0} \pm 1$ particles are degenerate up to the hybridization energy, which decays exponentially with the system size.

Importantly, our system is different from that hosting conventional Majorana states in both ways discussed above. First, it does not have a gap stemming from the pairing, and consequently the bulk remains gapless. As a result, the Majorana-like resonances do not have an exponential localization at the edge but rather power-law confinement. Thus, the hybridization energy decays as a power law with the system size. Second, we are considering a finite system which cannot exchange particles with an infinite superconductor, so that adding particles to the system costs energy $\propto 1 / L$, and therefore a splitting $\omega_{p} \propto 1 / L$ would be obtained independently on the type of localization of the edge modes (see also Sec. V). Both of these contributions are fully included in our calculations.

The dependence of the scaling coefficient $C(V)$ on the interaction strength is shown in Fig. 3(b). We observe that it decays almost linearly to zero in the vicinity of the critical point $V=2 t$.

\section{ROBUSTNESS OF THE ZERO MODES TO PERTURBATIONS}

Robustness against perturbations in the Hamiltonian is a paradigmatic property of topological states in general, and Majorana bound states in particular. To this end, we study the robustness of the peaks with respect to perturbations that break the integrability of the model. We consider three different types of perturbations: second-neighbor hopping, second-neighbor interactions, and onsite disorder. These perturbations are described by the following Hamiltonians, respectively:

$$
\begin{gathered}
\hat{H}_{2 \mathrm{nh}}=-t^{\prime} \sum_{j=1}^{L-2}\left(\hat{c}_{j+2}^{\dagger} \hat{c}_{j}+\hat{c}_{j}^{\dagger} \hat{c}_{j+2}\right), \\
\hat{H}_{2 \mathrm{ni}}=-V^{\prime} \sum_{j=1}^{L-2}\left(\hat{c}_{j+2}^{\dagger} \hat{c}_{j+2}-\frac{1}{2}\right)\left(\hat{c}_{j}^{\dagger} \hat{c}_{j}-\frac{1}{2}\right), \\
\hat{H}_{d}=\sum_{j=1}^{L} u_{j} \hat{c}_{j}^{\dagger} \hat{c}_{j},
\end{gathered}
$$

where $t^{\prime}$ and $V^{\prime}$ are the parameters determining the strengths of the second-neighbor hopping and interactions, respectively, and $u_{j}$ is the onsite potential chosen randomly in the range $[-W, W]$. The distributions of the local density of states for these types of perturbations are shown in Fig. 4. In all cases considered here, we find that weak perturbations do not affect the existence of the edge states.

\section{CONNECTION TO A TOPOLOGICAL SYMMETRY-BROKEN SUPERCONDUCTOR}

Above, we focused on the disordered quantum state, showing that the interacting gapless gas exhibits excitations sharing the robustness properties of topological Majorana zero modes. Although such similarity is suggestive of matching topological properties, a rigorous proof calls for a demonstration that
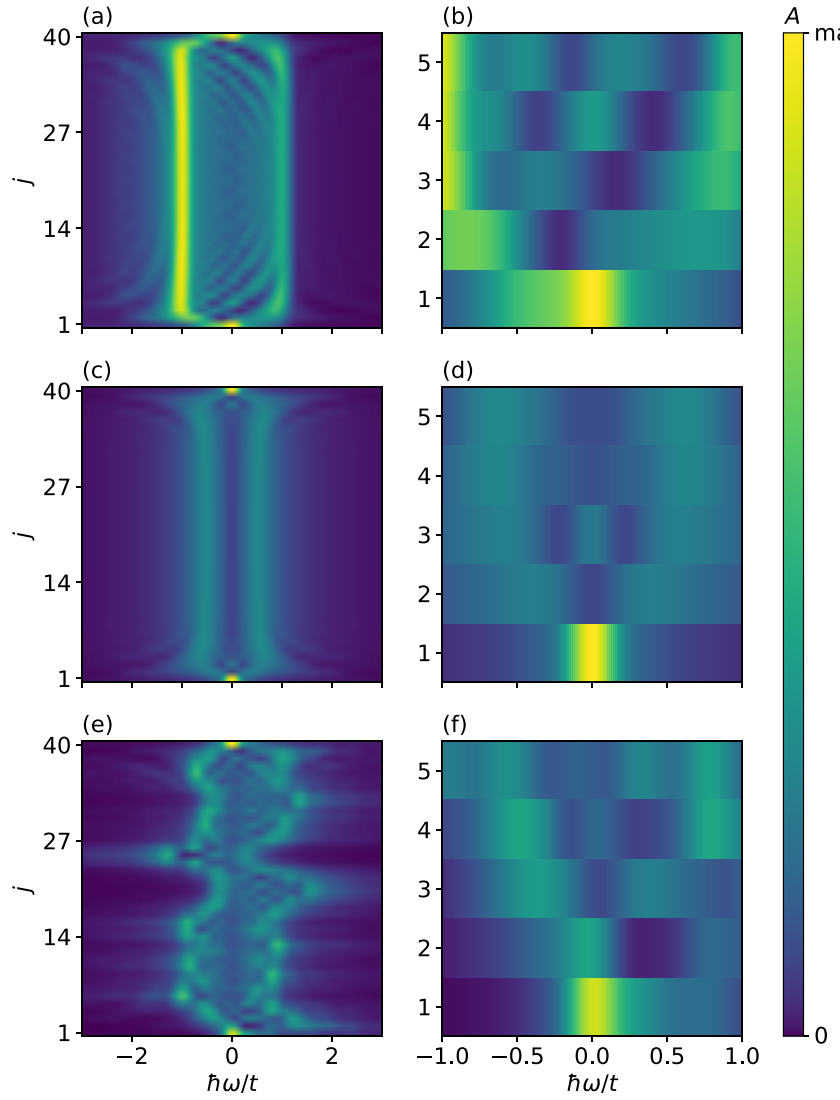

FIG. 4. Local density of states as a function of energy and the site index of the chain for perturbed systems with $L=40, V=$ $1.5 \times t, \mu=0$. The perturbations are (a), (b) second-neighbor hopping, (c), (d) second-neighbor interaction, and (e), (f) onsite disorder. The distinctive parameters are given by (a), (b) $t^{\prime}=-0.2 \times t$, (c), (d) $V^{\prime}=0.4 \times t$, (e), (f) $W=0.5 \times t$. In all cases, there are strong zero-energy modes at the edges demonstrating the robustness of these states.

the two types of excitations can be continuously transformed into each other. The aim of this section is to provide such a demonstration.

To this end, we introduce a family of Hamiltonians

$$
\hat{H}(s)=(1-s) \hat{H}_{I}+s \hat{H}_{K}-\tilde{\mu}(s) \hat{N}, \quad 0 \leqslant s \leqslant 1
$$

where $\hat{N}$ is the number of particle operators. This family yields a continuous transformation from the Hamiltonian of the interacting chain at $s=0$ to that of a topological superconductor with gauge symmetry breaking at $s=1$. Therefore, control of the parameter $s$ enables us to track the transformation from the Majorana-like modes without symmetry breaking to standard Majorana modes with symmetry breaking. The chemical potential $\tilde{\mu}(s)$ is used to control the total number of particles throughout the path.

The local density of states at the edge of the chain is shown in Fig. 5 as a function of the transformation parameter $s$. If $|\mu|<2 t-V$ and the chemical potential does not depend on the parameter $\tilde{\mu}(s)=0$, the bulk gap exactly closes at $s=0$, as shown in Fig. 5(a). Another way to connect the fully interacting model to the Kitaev chain is to keep the mean number of particles $\langle\hat{N}\rangle(s)$ constant and sweep the parameter 

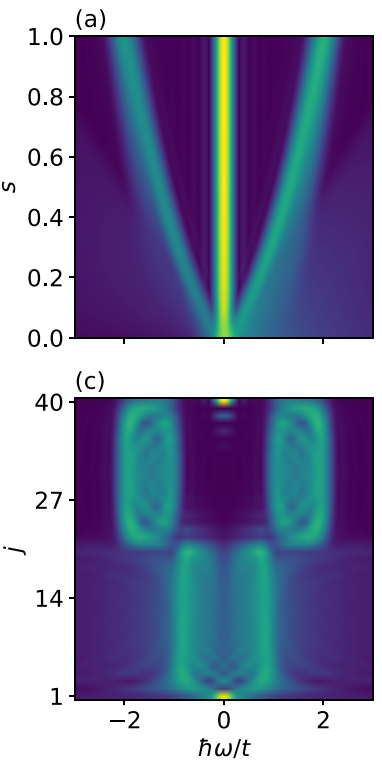

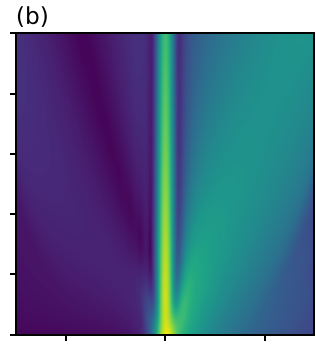

(d)

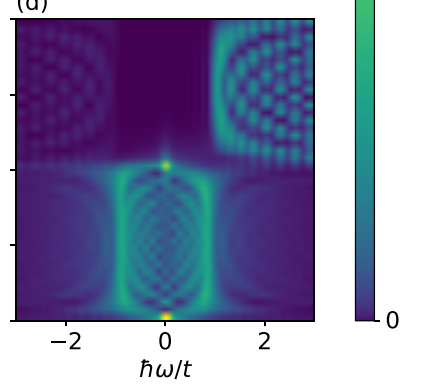

FIG. 5. (a), (b) Local density of states at the first site as a function of the transformation parameter $s$ and energy calculated for the Hamiltonian (7) with $L=40, V=1.5 \times t$, and $\Delta=t$. In (a), we use $\mu=-0.2 \times t$ and $\tilde{\mu}(s)=0$, whereas in (b), $\tilde{\mu}(s)$ is adjusted in such a way that $\langle\hat{N}\rangle(s) / L=1 / 4$. (c), (d) Local density of states for each site in a heterojunction, described by the Hamiltonian (8), between a topological superconductor and the interacting model (c), and between a trivial superconductor and the interacting model (d). In both panels, the lower half of the system shows data for the interacting model. The parameters are set to $L^{\prime}=20, \Delta=0.5 \times t$, and $\mu=0$ in both panels and to (c) $\mu^{\prime}=0$ and (d) $\mu^{\prime}=3$.

$s$. This case is shown in Fig. 5(b) yielding qualitatively similar results to those with a constant chemical potential.

We emphasize that the above-discovered connection between the Majorana-like modes and the symmetry-broken Majorana zero modes in topological superconductors has important consequences. In particular, similar to Majorana zero modes, the Majorana-like modes in non-superconducting states appear as a conductance peak at zero voltage bias if probed with a scanning tunnel microscope at the edge of the chain, a phenomenon referred to as zero-bias anomaly. Thus, both modes have similar signatures in typical experiments. These states will, however, coexist with a gapless background of edge excitations in the bulk. In addition, the gapless nature of the bulk excitations may give rise to decoherence of the phase information in these Majorana-like modes different from that in conventional Majorana bound states [78-80].

The equivalence between the zero modes of the interacting model and the conventional Majorana zero modes can be further emphasized by studying a heterojunction between the interacting model and a topological or trivial superconductor. The model Hamiltonian of such a heterojunction between sites $L^{\prime}$ and $L^{\prime}+1$ has the following form:

$$
\hat{H}=-t \sum_{j=1}^{L-1}\left(\hat{c}_{j+1}^{\dagger} \hat{c}_{j}+\hat{c}_{j}^{\dagger} \hat{c}_{j+1}\right)-\mu \sum_{j=1}^{L^{\prime}} \hat{c}_{j}^{\dagger} \hat{c}_{j}
$$

$$
\begin{aligned}
& -V \sum_{j=1}^{L^{\prime}-1}\left(\hat{c}_{j+1}^{\dagger} \hat{c}_{j+1}-\frac{1}{2}\right)\left(\hat{c}_{j}^{\dagger} \hat{c}_{j}-\frac{1}{2}\right) \\
& +\Delta \sum_{j=L^{\prime}}^{L-1}\left(\hat{c}_{j+1}^{\dagger} \hat{c}_{j}^{\dagger}+\hat{c}_{j} \hat{c}_{j+1}\right)-\mu^{\prime} \sum_{j=L^{\prime}+1}^{L} \hat{c}_{j}^{\dagger} \hat{c}_{j},
\end{aligned}
$$

where $\Delta$ and $\mu^{\prime}$ are the superconducting order parameter and the chemical potential in the topological superconductor, respectively. Here $\left|\mu^{\prime}\right|<2 t$ corresponds to the topological superconductor and $\left|\mu^{\prime}\right|>2 t$ to the trivial one.

The local density of states in the case of the heterojunction is shown in Figs. 5(c) and 5(d). For an interface between the interacting model and a topological superconductor, no resonance is expected at the junction since the Majorana zero mode of the topological superconductor and the Majorana-like mode of the interacting model annihilate each other as shown in Fig. 5(c). In contrast, for the interface between the interacting model and a conventional superconductor, a zero mode remains at the junction as is clearly visible in Fig. 5(d). Note that a similar phenomenon would be observed if the interacting model were replaced by a symmetry-broken topological superconductor.

\section{BOSONIZED CONTINUUM LIMIT}

In order to show that the discovered zero-energy peaks exist for an arbitrarily weak attractive interaction, we employ a bosonization technique for a continuous analog of our model, described by the following Hamiltonian:

$$
\hat{H}_{c}=\int_{0}^{L}\left\{\hat{\psi}^{\dagger}\left(-\frac{\hbar^{2} \partial_{x}^{2}}{2 m}-\frac{\hbar^{2} k_{F}^{2}}{2 m}\right) \hat{\psi}+\frac{g}{8 k_{F}^{2}}:\left[\partial_{x} \hat{\rho}\right]^{2}:\right\} d x,
$$

where $\hat{\psi}^{\dagger}$ and $\hat{\psi}$ are the fermionic creation and annihilation operators, respectively, $m$ is the mass of the fermion, $\hbar k_{F}$ is the Fermi momentum, $g$ is the interaction parameter, $\hat{\rho}=$ $\hat{\psi}^{\dagger} \hat{\psi}$ is the density operator, and :...: denotes the normal ordering of the operator. Here, we impose zero boundary conditions $\hat{\psi}(0)=\hat{\psi}(L)=0$. Following Ref. [81], we introduce an auxiliary right-moving field $\hat{\psi}_{R}(x)$ defined on the segment $[-L, L]$ as

$$
\hat{\psi}(x)=e^{i k_{F} x} \hat{\psi}_{R}(x)-e^{-i k_{F} x} \hat{\psi}_{R}(-x) .
$$

Zero boundary conditions for $\hat{\psi}(x)$ are equivalent to periodic ones for $\hat{\psi}_{R}(x)$ and, hence, the latter field can be straightforwardly bosonized. The Hamiltonian expressed in terms of $\hat{\psi}_{R}$ has the following form:

$$
\hat{H}_{c} \approx \int_{-L}^{L}\left[-i \hbar v_{F} \hat{\psi}_{R}^{\dagger} \partial_{x} \hat{\psi}_{R}-\frac{g}{2}: \hat{\rho}_{R}(x) \hat{\rho}_{R}(-x):\right] d x,
$$

where $v_{F}=\hbar k_{F} / m, \hat{\rho}_{R}=\psi_{R}^{\dagger} \psi_{R}$, and we have replaced the quadratic dispersion by a linear one in the vicinity of the Fermi surface. The bosonization expression for the field $\hat{\psi}_{R}(x)$ reads as [82]

$$
\hat{\psi}_{R}(x)=\frac{\hat{F}}{\sqrt{2 \pi a}} e^{i \pi \hat{n} x / L} e^{i \hat{\phi}(x)},
$$

where $\hat{n}$ is a number operator for excess particles with respect to the Fermi sea state, i.e., the ground state of a noninteracting 
wire with $g=0$, and $\hat{F}$ is the Klein factor $[\hat{n}, \hat{F}]=-\hat{F}$, $\hat{F}^{\dagger} \hat{F}=\hat{F} \hat{F}^{\dagger}=1$. The phase field is given by the following expression:

$$
\hat{\phi}(x)=i \sum_{k>0} \sqrt{\frac{\pi}{k L}}\left(e^{-i k x} \hat{b}_{k}^{\dagger}-e^{i k x} \hat{b}_{k}\right) e^{-k a / 2},
$$

where $k=\pi j / L, j$ is a positive integer, $\hat{b}_{k}$ and $\hat{b}_{k}^{\dagger}$ are the bosonic annihilation and creation operators for the mode $k$, respectively, and we have introduced a regularization parameter $a$ such that all the expressions are to be taken in the limit $a \rightarrow+0$. In terms of the bosonic operators the Hamiltonian is expressed as

$$
\begin{aligned}
\hat{H}_{c}= & \sum_{k>0} k\left[\hbar v_{F} \hat{b}_{k}^{\dagger} \hat{b}_{k}-\frac{g}{4 \pi}\left(\hat{b}_{k} \hat{b}_{k}+\hat{b}_{k}^{\dagger} \hat{b}_{k}^{\dagger}\right)\right] \\
& +\frac{\pi}{2 L}\left(\hbar v_{F}-\frac{g}{2 \pi}\right) \hat{n}^{2} .
\end{aligned}
$$

This Hamiltonian can be diagonalized by the Bogoliubov transformation

$$
\hat{b}_{k}=\tilde{b}_{k} \cosh \varphi+\tilde{b}_{k}^{\dagger} \sinh \varphi, \quad \tanh 2 \varphi=\frac{g}{2 \pi \hbar v_{F}},
$$

and reduced to the following form:

$$
\hat{H}_{c}=\sum_{k>0} k \sqrt{\hbar^{2} v_{F}^{2}-\left(\frac{g}{2 \pi}\right)^{2}} \tilde{b}_{k}^{\dagger} \tilde{b}_{k}+\frac{\pi}{2 L}\left(\hbar v_{F}-\frac{g}{2 \pi}\right) \hat{n}^{2} .
$$

We note that the Bogoliubov transformation requires $|g|<$ $2 \pi \hbar v_{F}$ since otherwise the Hamiltonian (14) is not bounded from below. Note that the case of strong interactions corresponds to the phase separation or charge density wave which are not accurately described by this bosonization formalism.

The local density of states of the bosonized wire is given by

$$
A(x, \omega)=i \int_{-\infty}^{\infty}\left[G^{>}(x, x, t)-G^{<}(x, x, t)\right] e^{i \omega t} d t,
$$

where

$$
\begin{aligned}
& G^{>}\left(x, x^{\prime}, t\right)=-i\left\langle\hat{\psi}(x, t) \hat{\psi}^{\dagger}\left(x^{\prime}, 0\right)\right\rangle \\
& \approx G_{R}^{>}\left(x, x^{\prime}, t\right)+G_{R}^{>}\left(-x,-x^{\prime}, t\right), \\
& G_{R}^{>}\left(x, x^{\prime}, t\right)=-i\left\langle\hat{\psi}_{R}(x, t) \hat{\psi}_{R}^{\dagger}\left(x^{\prime}, 0\right)\right\rangle, \\
& G^{<}\left(x, x^{\prime}, t\right)=i\left\langle\hat{\psi}^{\dagger}\left(x^{\prime}, 0\right) \hat{\psi}(x, t)\right\rangle \\
& \approx G_{R}^{<}\left(x, x^{\prime}, t\right)+G_{R}^{<}\left(-x,-x^{\prime}, t\right),
\end{aligned}
$$

and

$$
G_{R}^{<}\left(x, x^{\prime}, t\right)=i\left\langle\hat{\psi}_{R}^{\dagger}\left(x^{\prime}, 0\right) \hat{\psi}_{R}(x, t)\right\rangle .
$$

On the right side of Eqs. (18) and (20) we have neglected the terms oscillating as $e^{ \pm 2 k_{F}\left(x+x^{\prime}\right)}$. The Green's function of the field $\hat{\psi}_{R}$ can be evaluated analytically (see Appendix B for details). Figure 6 shows the local density of states of the wire as a function of the coordinate and energy. We clearly observe zero-energy peaks at the ends of the wire.

The form of the Hamiltonian in Eq. (16) allows us to analytically evaluate the gap in the local density of states.
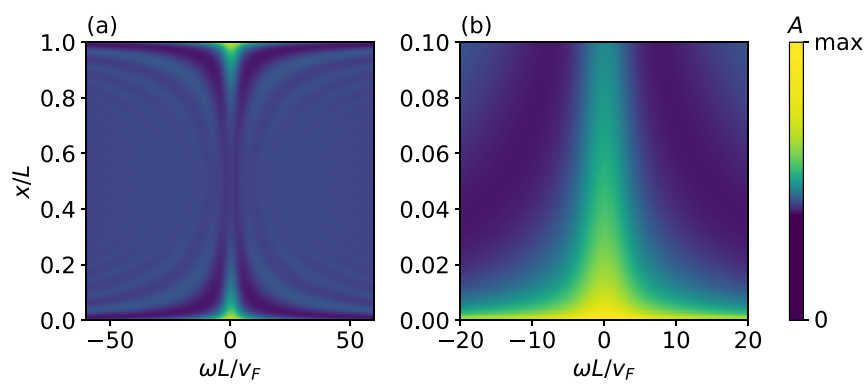

FIG. 6. (a), (b) Local density of states $A(x, \omega)$ for the continuous wire of interacting fermions normalized by the local density of states of the noninteracting system as a function of the position $x$ and energy $\omega$. (b) Shows data close to the end of the wire. Here, $\hbar v_{F}=g$ and the energy broadening of the local density of states equals $\delta=\hbar v_{F} /(2 L)$.

This gap is equal to $\pi /(2 L)\left[\hbar v_{F}-g /(2 \pi)\right]$ since it is the lowest energy needed to add or remove a single particle to or from the wire without exciting any of the bosonic modes. This expression is in the good qualitative agreement with the results shown in Fig. 3. Namely, the peak splitting scales as $C / L$ and the factor $C$ linearly decays with increasing interaction strength up to the point of transition to phase separation.

\section{CONCLUSIONS}

In summary, we demonstrated the emergence of zero-bias modes at the edges of a 1D chain of attractively interacting fermions. We showed that these modes can be continuously transformed to conventional Majorana zero modes of a topological superconductor, yet with the striking difference that they emerge in the absence gauge symmetry breaking. These many-body zero modes are found to exist at arbitrarily weak attractive interaction and are robust to perturbations which break the integrability of the model, including longrange hopping and interactions, and disorder. In addition, we demonstrated that these zero-mode resonances can be analyzed with both a lattice quantum many-body formalism based on tensor networks and a continuum low-energy model based on bosonization. Our results introduce an unexpected type of protected zero modes in a pure many-body case, with no single-particle analog, providing a stepping stone towards the exploration of topological modes in generic disordered quantum many-body systems.

However, despite the demonstration of the robustness of the many-body zero modes with respect to integrability breaking perturbations, it is not clear whether these modes have truly topological origin, i.e., can be rationalized by introducing some topological invariant. It must be noted though that the topological classifications for interacting systems have, in general, not been understood up to date. We hope that our results of the existence of these zero modes will inspire work in this direction, and motivate future studies addressing the topological invariant for such many-body system. Another question we do not consider in this work is implications for the quantum computing. The gapless nature of the system and power-law localization of the observed zero modes are disadvantageous for any practical purposes. However, the effects 
detrimental for conventional Majorana-based computing like dephasing or quasiparticle poisoning may be less pronounced in the considered many-body system. This issue should also be addressed in future researches.

\section{ACKNOWLEDGMENTS}

We acknowledge the financial support from our Academy of Finland projects (Grants No. 331342 and No. 336243) and its Centre of Excellence in Quantum Technology (QTF) (Grant No. 336810), and from the European Research Council under Grant No. 681311 (QUESS). The research was also partially supported by the Foundation for Polish Science through the IRA Programme co-financed by EU within SG OP. We thank S. Sharov and P. Nosov for useful discussions and the Aalto Science-IT project for computational resources.

\section{APPENDIX A: CRITICAL POINT}

In the general interacting case the Bethe ansatz expression for the ground state of the system is complicated except for the critical point $\mu=0, V=2 t$. In this case the ground state has the form [83]

$$
\left|\Psi_{0}\right\rangle=\frac{1}{2^{L / 2}} \prod_{j=1}^{L}\left(1+\hat{c}_{j}^{\dagger}\right)|0\rangle,
$$

corresponding to energy $E_{0}=t(1-L) / 2$. Interestingly, this is actually the same ground state as in the Kitaev model, and thus it would be possible to construct Majorana operators describing localized zero-energy excitations [84]. It must be noted that in the present model there will be also additional low-energy excitations as we are considering a truly interacting model instead of mean-field Hamiltonian.

In order to prove this result we define $\left|\Psi_{j-1}\right\rangle$ be defined as

$$
\left|\Psi_{j-1}\right\rangle=\frac{1}{2^{L / 2}} \prod_{k=j}^{L}\left(1+\hat{c}_{k}^{\dagger}\right)|0\rangle
$$

At the same time we define $\hat{H}_{j-1}$ as

$$
\begin{aligned}
\hat{H}_{j-1}= & -t \sum_{k=j}^{L-1}\left(\hat{c}_{k+1}^{\dagger} \hat{c}_{k}+\hat{c}_{k}^{\dagger} \hat{c}_{k+1}\right) \\
& -2 t \sum_{k=j}^{L-1}\left(\hat{\hat{c}}_{k+1}^{\dagger} \hat{c}_{k+1}-\frac{1}{2}\right)\left(\hat{c}_{k}^{\dagger} \hat{\hat{c}}_{k}-\frac{1}{2}\right) .
\end{aligned}
$$

It is obvious that $\hat{H}_{0}=\hat{H}_{I}$ at $V=2 t$ and $\mu=0$. We are going to prove that $\left|\Psi_{j-1}\right\rangle$ is the eigenstate of the Hamiltonian $\hat{H}_{j-1}$ with the energy $t(j-L) / 2$. For $j=L$ this fact is obvious because $\hat{H}_{L-1}=0$. Assume that for some $j-1$ this statement is true. Then let us prove it for $j-2$ :

$$
\begin{aligned}
\hat{H}_{j-2}\left|\Psi_{j-2}\right\rangle & =\left[-t\left(\hat{c}_{j}^{\dagger} \hat{c}_{j-1}+\hat{c}_{j-1}^{\dagger} \hat{c}_{j}\right)-2 t\left(\hat{c}_{j}^{\dagger} \hat{\hat{c}}_{j}-\frac{1}{2}\right)\left(\hat{c}_{j-1}^{\dagger} \hat{c}_{j-1}-\frac{1}{2}\right)+\hat{H}_{j}\right]\left(1+\hat{c}_{j-1}^{\dagger}\right)\left|\Psi_{j-1}\right\rangle \\
& =\frac{t(j-L)}{2}\left|\Psi_{j-2}\right\rangle-t\left\{\hat{c}_{j-1}^{\dagger}\left|\Psi_{j}\right\rangle-\left[\left(\hat{c}_{j}^{\dagger} \hat{\hat{c}}_{j}-\frac{1}{2}\right)+\hat{c}_{j}^{\dagger} \hat{c}_{j-1} \hat{c}_{j-1}^{\dagger}+2\left(\hat{c}_{j}^{\dagger} \hat{\hat{c}}_{j}-\frac{1}{2}\right)\left(\hat{c}_{j-1}^{\dagger} \hat{c}_{j-1}-\frac{1}{2}\right) \hat{c}_{j-1}^{\dagger}\right]\left|\Psi_{j-1}\right\rangle\right\} \\
& =\frac{t(j-L)}{2}\left|\Psi_{j-2}\right\rangle-t\left[\hat{c}_{j-1}^{\dagger}\left|\Psi_{j}\right\rangle-\hat{c}_{j}^{\dagger}\left|\Psi_{j}\right\rangle+\frac{1}{2}\left|\Psi_{j-1}\right\rangle+\hat{c}_{j}^{\dagger}\left|\Psi_{j}\right\rangle+\left(\hat{c}_{j}^{\dagger} \hat{c}_{j}-\frac{1}{2}\right) \hat{c}_{j-1}^{\dagger}\left|\Psi_{j-1}\right\rangle\right] \\
& =\frac{t(j-L)}{2}\left|\Psi_{j-2}\right\rangle-t\left[\hat{c}_{j-1}^{\dagger}\left|\Psi_{j}\right\rangle+\frac{1}{2}\left|\Psi_{j-1}\right\rangle+\hat{c}_{j-1}^{\dagger} \hat{c}_{j}^{\dagger}\left|\Psi_{j}\right\rangle-\frac{1}{2} \hat{c}_{j-1}^{\dagger}\left|\Psi_{j-1}\right\rangle\right] \\
& =\frac{t(j-L)}{2}\left|\Psi_{j-2}\right\rangle-t\left[\frac{1}{2}\left|\Psi_{j-1}\right\rangle+\frac{1}{2} \hat{c}_{j-1}^{\dagger}\left|\Psi_{j-1}\right\rangle\right]=\frac{t(j-1-L)}{2}\left|\Psi_{j-2}\right\rangle .
\end{aligned}
$$

In order to prove that the found eigenstate is the ground state, i.e., has the minimal possible energy, we notice that the Hamiltonian can be written as

$$
\hat{H}_{I}=-t \sum_{j=1}^{L-1}\left[\hat{c}_{j+1}^{\dagger} \hat{c}_{j}+\hat{c}_{j}^{\dagger} \hat{c}_{j+1}+2\left(\hat{c}_{j+1}^{\dagger} \hat{c}_{j+1}-\frac{1}{2}\right)\left(\hat{c}_{j}^{\dagger} \hat{c}_{j}-\frac{1}{2}\right)\right]
$$

Each term of the sum has the lowest eigenvalue equal to $-t / 2$ and there are $L-1$ terms total, then the energy of the ground state cannot be lower than $t(1-L) / 2$.

Since the state $\left|\Psi_{0}\right\rangle$ is not an eigenstate of the number of particle operators, one can project it onto the subspaces with the fixed number of particles yielding $L+1$-times degenerate ground states corresponding to the different number of particles $N$ from 0 to $L$ :

$$
\left|\Psi_{0 N}\right\rangle=\frac{2^{L / 2}}{\sqrt{\left(\begin{array}{c}
L \\
N
\end{array}\right)}} \hat{P}_{N}\left|\Psi_{0}\right\rangle
$$

where $\hat{P}_{N}$ is an orthogonal projector onto the subspace with $N$ particles. 


\section{APPENDIX B: GREEN'S FUNCTIONS OF THE CONTINUOUS WIRE}

The greater and lesser Green's functions of the field $\hat{\psi}_{R}$ are equal to

$$
\begin{gathered}
G_{R}^{>}\left(x, x^{\prime}, t\right)=-\frac{i}{2 \pi a}\left\langle\hat{F}(t) e^{i \pi \hat{n}\left(x-x^{\prime}\right) / L} \hat{F}^{\dagger} e^{i \hat{\phi}(x, t)} e^{-i \hat{\phi}\left(x^{\prime}\right)}\right\rangle, \\
G_{R}^{<}\left(x, x^{\prime}, t\right)=\frac{i}{2 \pi a}\left\langle e^{-i \pi \hat{n} x^{\prime} / L} \hat{F}^{\dagger} \hat{F}(t) e^{i \pi \hat{n} x / L} e^{-i \hat{\phi}\left(x^{\prime}\right)} e^{i \hat{\phi}(x, t)}\right\rangle,
\end{gathered}
$$

where $\hat{F}(t)=e^{i \hat{H}_{c} t / \hbar} \hat{F} e^{-i \hat{H}_{c} t / \hbar}$ and $\hat{\phi}(x, t)=e^{i \hat{H}_{c} t / \hbar} \hat{\phi}(x) e^{-i \hat{H}_{c} t / \hbar}$. After long but straightforward calculations one obtains

$$
\begin{aligned}
G_{R}^{>}\left(x, x^{\prime}, t\right)= & -\frac{i c}{2 L} \exp \left[-\frac{i \pi t}{2 L}\left(v_{F}-\frac{g}{2 \pi \hbar}\right)+\frac{i \pi\left(x-x^{\prime}\right)}{L}\right] \\
& \times \frac{\left[1-e^{-\frac{\pi}{L}\left(a+i \tilde{v}_{F} t+i x+i x^{\prime}\right)}\right]^{\frac{s}{2}}\left[1-e^{-\frac{\pi}{L}\left(a+i \tilde{v}_{F} t-i x-i x^{\prime}\right)}\right]^{\frac{s}{2}}\left[1-e^{-\frac{\pi}{L}\left(a+i \tilde{v}_{F} t+i x-i x^{\prime}\right)}\right]^{\frac{1-c}{2}}}{\left|1-e^{-\frac{\pi}{L}(a-2 i x)}\right|^{\frac{s}{2}} \mid 1-e^{-\left.\frac{\pi}{L}\left(a-2 i x^{\prime}\right)\right|^{\frac{s}{2}}}\left[1-e^{-\frac{\pi}{L}\left(a+i \tilde{v}_{F} t-i x+i x^{\prime}\right)}\right]^{\frac{1+c}{2}}}, \\
G_{R}^{<}\left(x, x^{\prime}, t\right)= & \frac{i c}{2 L} \exp \left[\frac{i \pi t}{2 L}\left(v_{F}-\frac{g}{2 \pi \hbar}\right)\right] \\
& \times \frac{\left[1-e^{-\frac{\pi}{L}\left(a-i \tilde{v}_{F} t-i x-i x^{\prime}\right)}\right]^{\frac{s}{2}}\left[1-e^{-\frac{\pi}{L}\left(a-i \tilde{v}_{F} t+i x+i x^{\prime}\right)}\right]^{\frac{s}{2}}\left[1-e^{-\frac{\pi}{L}\left(a-i \tilde{v}_{F} t-i x+i x^{\prime}\right)}\right]^{\frac{1-c}{2}}}{\left|1-e^{-\frac{\pi}{L}(a+2 i x)}\right|^{\frac{s}{2}} \mid 1-e^{-\left.\frac{\pi}{L}\left(a+2 i x^{\prime}\right)\right|^{\frac{s}{2}}}\left[1-e^{-\frac{\pi}{L}\left(a-i \tilde{v}_{F} t+i x-i x^{\prime}\right)}\right]^{\frac{1+c}{2}}},
\end{aligned}
$$

where $c=\cosh 2 \varphi, s=\sinh 2 \varphi$, and $\tilde{v}_{F}=\sqrt{v_{F}^{2}-g^{2} /(2 \pi \hbar)^{2}}$. Finally, the expression for the local density of states reads as

$$
A(x, \omega)=2 \int_{-\infty}^{+\infty} e^{i \omega t} \operatorname{Re}\left\{e^{-\frac{i \pi t}{2 L}\left(v_{F}-\frac{g}{2 \pi \hbar}\right)} \frac{\left(1-e^{-\frac{\pi a}{L}}\right)^{c}\left[1-e^{-\frac{\pi}{L}\left(a-i \tilde{v}_{F} t-2 i x\right)}\right]^{\frac{s}{2}}\left[1-e^{-\frac{\pi}{L}\left(a-i \tilde{v}_{F} t+2 i x\right)}\right]^{\frac{s}{2}}}{\pi a\left|1-e^{-\frac{\pi}{L}(a+2 i x)}\right|^{s}\left[1-e^{-\frac{\pi}{L}\left(a-i v_{F} t\right)}\right]^{c}}\right\} d t .
$$

\section{APPENDIX C: PERSISTENT CURRENT IN A RING}

We close up the chain into a ring with a weak link and pierce it with a magnetic flux. The Hamiltonian of such a
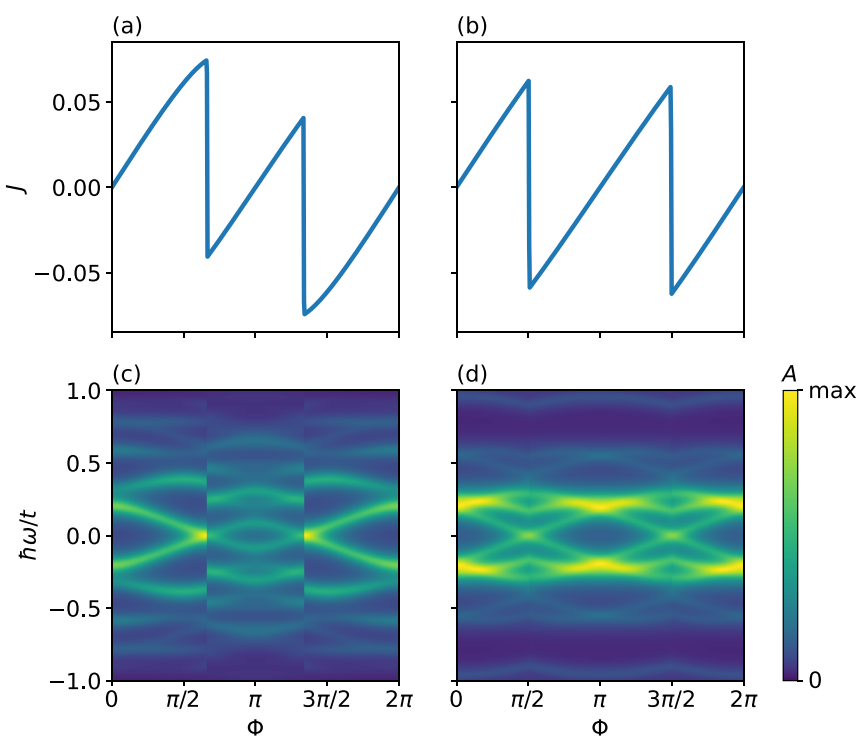

FIG. 7. (a), (b) Persistent current in the ring as a function of flux. (c), (d) The local density of states at the site adjacent to the weak link as a function of energy and flux. The parameters are $t_{w}=0.2 \times t$, $L=10, \mu=0$, (a), (c) $V=1.5 \times t$, (b), (d) $V=2 t$. The data are obtained using an exact diagonalization of the Hamiltonian (C1). The energy broadening of the local density of states equals $\delta=0.05 \times t$. system reads as

$$
\hat{H}_{I}^{\prime}=\hat{H}_{I}-t_{w}\left(\hat{c}_{L}^{\dagger} \hat{c}_{1} e^{i \Phi}+\hat{c}_{1}^{\dagger} \hat{c}_{L} e^{-i \Phi}\right),
$$

where $t_{w}$ is hopping parameter through the weak link between the first and the last sites of the chain, and $\Phi$ determines the flux in the units of $\Phi_{0} /(2 \pi)$, where $\Phi_{0}$ is the normal (not superconducting) flux quantum. The current operator is defined as

$$
\hat{J}=\frac{\partial \hat{H}_{I}^{\prime}}{\partial \Phi}=-i t_{w}\left(\hat{c}_{L}^{\dagger} \hat{c}_{1} e^{i \Phi}-\hat{c}_{1}^{\dagger} \hat{c}_{L} e^{-i \Phi}\right)
$$

The current dependence on the flux is shown in Figs. 7(a) and 7(b) for different values of interaction strength. The discontinuities in the current-flux relation correspond to the number of particle switches in the ring. Figures 7(c) and 7(d) show the local density of states at the site adjacent to the weak link as a function of flux and energy. The peak splitting oscillates as a function of flux, the number of particle switches take place exactly at the peak's intersections. The behavior of the current and spectral function at the critical point $V=2 t$ mostly resembles the behavior of the mean field Kitaev model where these quantities are $\pi$ periodic with the flux if one allows parity switches, and $2 \pi$ periodic if the parity is kept fixed. Away from the critical point we do not observe exact $\pi$ periodicity of the current in the presence of parity switches which may be a consequence of a finite overlap of the peaks through the bulk of the chain. 
[1] X.-L. Qi and S.-C. Zhang, Topological insulators and superconductors, Rev. Mod. Phys. 83, 1057 (2011).

[2] M. Sato and Y. Ando, Topological superconductors: A review, Rep. Prog. Phys. 80, 076501 (2017).

[3] S. R. Elliott and M. Franz, Colloquium: Majorana fermions in nuclear, particle, and solid-state physics, Rev. Mod. Phys. 87, 137 (2015).

[4] D. A. Ivanov, Non-Abelian Statistics of Half-Quantum Vortices in $p$-Wave Superconductors, Phys. Rev. Lett. 86, 268 (2001).

[5] A. Y. Kitaev, Fault-tolerant quantum computation by anyons, Ann. Phys. 303, 2 (2003).

[6] C. Nayak, S. H. Simon, A. Stern, M. Freedman, and S. Das Sarma, Non-abelian anyons and topological quantum computation, Rev. Mod. Phys. 80, 1083 (2008).

[7] M. Sato, Y. Takahashi, and S. Fujimoto, Non-Abelian Topological Order in $s$-Wave Superfluids of Ultracold Fermionic Atoms, Phys. Rev. Lett. 103, 020401 (2009).

[8] J. D. Sau, R. M. Lutchyn, S. Tewari, and S. Das Sarma, Generic New Platform for Topological Quantum Computation using Semiconductor Heterostructures, Phys. Rev. Lett. 104, 040502 (2010).

[9] J. D. Sau, S. Tewari, R. M. Lutchyn, T. D. Stanescu, and S. Das Sarma, Non-abelian quantum order in spin-orbit-coupled semiconductors: Search for topological majorana particles in solid-state systems, Phys. Rev. B 82, 214509 (2010).

[10] T. Hyart, B. van Heck, I. C. Fulga, M. Burrello, A. R. Akhmerov, and C. W. J. Beenakker, Flux-controlled quantum computation with Majorana fermions, Phys. Rev. B 88, 035121 (2013).

[11] T. Karzig, C. Knapp, R. M. Lutchyn, P. Bonderson, M. B. Hastings, C. Nayak, J. Alicea, K. Flensberg, S. Plugge, Y. Oreg, C. M. Marcus, and M. H. Freedman, Scalable designs for quasiparticle-poisoning-protected topological quantum computation with Majorana zero modes, Phys. Rev. B 95, 235305 (2017).

[12] R. M. Lutchyn, E. P. A. M. Bakkers, L. P. Kouwenhoven, P. Krogstrup, C. M. Marcus, and Y. Oreg, Majorana zero modes in superconductor-semiconductor heterostructures, Nat. Rev. Mater. 3, 52 (2018).

[13] C. W. J. Beenakker, Search for non-Abelian Majorana braiding statistics in superconductors, SciPost Phys. Lect. Notes 15 (2020).

[14] R. M. Lutchyn, J. D. Sau, and S. Das Sarma, Majorana Fermions and a Topological Phase Transition in Semiconductor-Superconductor Heterostructures, Phys. Rev. Lett. 105, 077001 (2010).

[15] Y. Oreg, G. Refael, and F. von Oppen, Helical Liquids and Majorana Bound States in Quantum Wires, Phys. Rev. Lett. 105, 177002 (2010).

[16] T. D. Stanescu, R. M. Lutchyn, and S. Das Sarma, Majorana fermions in semiconductor nanowires, Phys. Rev. B 84, 144522 (2011).

[17] V. Mourik, K. Zuo, S. M. Frolov, S. R. Plissard, E. P. A. M. Bakkers, and L. P. Kouwenhoven, Signatures of Majorana fermions in hybrid superconductor-semiconductor nanowire devices, Science 336, 1003 (2012).

[18] A. D. K. Finck, D. J. Van Harlingen, P. K. Mohseni, K. Jung, and X. Li, Anomalous Modulation of a Zero-Bias Peak in a Hybrid Nanowire-Superconductor Device, Phys. Rev. Lett. 110, 126406 (2013).
[19] H. O. H. Churchill, V. Fatemi, K. Grove-Rasmussen, M. T. Deng, P. Caroff, H. Q. Xu, and C. M. Marcus, Superconductornanowire devices from tunneling to the multichannel regime: Zero-bias oscillations and magnetoconductance crossover, Phys. Rev. B 87, 241401(R) (2013).

[20] D. Rainis, L. Trifunovic, J. Klinovaja, and D. Loss, Towards a realistic transport modeling in a superconducting nanowire with Majorana fermions, Phys. Rev. B 87, 024515 (2013).

[21] A. E. Antipov, A. Bargerbos, G. W. Winkler, B. Bauer, E. Rossi, and R. M. Lutchyn, Effects of Gate-Induced Electric Fields on Semiconductor Majorana Nanowires, Phys. Rev. X 8, 031041 (2018).

[22] T. Karzig, G. Refael, and F. von Oppen, Boosting Majorana Zero Modes, Phys. Rev. X 3, 041017 (2013).

[23] T.-P. Choy, J. M. Edge, A. R. Akhmerov, and C. W. J. Beenakker, Majorana fermions emerging from magnetic nanoparticles on a superconductor without spin-orbit coupling, Phys. Rev. B 84, 195442 (2011).

[24] S. Nadj-Perge, I. K. Drozdov, B. A. Bernevig, and A. Yazdani, Proposal for realizing Majorana fermions in chains of magnetic atoms on a superconductor, Phys. Rev. B 88, 020407(R) (2013).

[25] S. Nadj-Perge, I. K. Drozdov, J. Li, H. Chen, S. Jeon, J. Seo, A. H. MacDonald, B. A. Bernevig, and A. Yazdani, Observation of Majorana fermions in ferromagnetic atomic chains on a superconductor, Science 346, 602 (2014).

[26] A. Heimes, P. Kotetes, and G. Schön, Majorana fermions from Shiba states in an antiferromagnetic chain on top of a superconductor, Phys. Rev. B 90, 060507(R) (2014).

[27] J. Klinovaja, P. Stano, A. Yazdani, and D. Loss, Topological Superconductivity and Majorana Fermions in Rkky Systems, Phys. Rev. Lett. 111, 186805 (2013).

[28] L. Fu and C. L. Kane, Superconducting Proximity Effect and Majorana Fermions at the Surface of a Topological Insulator, Phys. Rev. Lett. 100, 096407 (2008).

[29] L. Fu and C. L. Kane, Josephson current and noise at a superconductor/quantum-spin-Hall-insulator/superconductor junction, Phys. Rev. B 79, 161408(R) (2009).

[30] J. R. Williams, A. J. Bestwick, P. Gallagher, S. S. Hong, Y. Cui, A. S. Bleich, J. G. Analytis, I. R. Fisher, and D. Goldhaber-Gordon, Unconventional Josephson Effect in Hybrid Superconductor-Topological Insulator Devices, Phys. Rev. Lett. 109, 056803 (2012)

[31] S. Hart, H. Ren, T. Wagner, P. Leubner, M. Mühlbauer, C. Brüne, H. Buhmann, L. W. Molenkamp, and A. Yacoby, Induced superconductivity in the quantum spin Hall edge, Nat. Phys. 10, 638 (2014).

[32] V. S. Pribiag, A. J. A. Beukman, F. Qu, M. C. Cassidy, C. Charpentier, W. Wegscheider, and L. P. Kouwenhoven, Edge-mode superconductivity in a two-dimensional topological insulator, Nat. Nanotechnol. 10, 593 (2015).

[33] H. Ren, F. Pientka, S. Hart, A. T. Pierce, M. Kosowsky, L. Lunczer, R. Schlereth, B. Scharf, E. M. Hankiewicz, L. W. Molenkamp, B. I. Halperin, and A. Yacoby, Topological superconductivity in a phase-controlled Josephson junction, Nature (London) 569, 93 (2019).

[34] A. Fornieri, A. M. Whiticar, F. Setiawan, E. Portolés, A. C. C. Drachmann, A. Keselman, S. Gronin, C. Thomas, T. Wang, R. Kallaher, G. C. Gardner, E. Berg, M. J. Manfra, A. Stern, C. M. Marcus, and F. Nichele, Evidence of topological 
superconductivity in planar Josephson junctions, Nature (London) 569, 89 (2019).

[35] P. San-Jose, J. L. Lado, R. Aguado, F. Guinea, and J. FernándezRossier, Majorana Zero Modes in Graphene, Phys. Rev. X 5, 041042 (2015).

[36] Y. Li, M. Amado, T. Hyart, G. P. Mazur, V. Risinggård, T. Wagner, L. McKenzie-Sell, G. Kimbell, J. Wunderlich, J. Linder, and J. W. A. Robinson, Transition between canted antiferromagnetic and spin-polarized ferromagnetic quantum Hall states in graphene on a ferrimagnetic insulator, Phys. Rev. B 101, 241405(R) (2020).

[37] L. Veyrat, C. Déprez, A. Coissard, X. Li, F. Gay, K. Watanabe, T. Taniguchi, Z. Han, B. A. Piot, H. Sellier, and B. Sacépé Helical quantum Hall phase in graphene on SrTiO3, Science 367, 781 (2020).

[38] M. Ezawa, Antiferromagnetic Topological Superconductor and Electrically Controllable Majorana Fermions, Phys. Rev. Lett. 114, 056403 (2015).

[39] J. L. Lado and M. Sigrist, Two-Dimensional Topological Superconductivity with Antiferromagnetic Insulators, Phys. Rev. Lett. 121, 037002 (2018).

[40] C.-H. Hsu, P. Stano, J. Klinovaja, and D. Loss, Antiferromagnetic nuclear spin helix and topological superconductivity in ${ }^{13}$ C nanotubes, Phys. Rev. B 92, 235435 (2015).

[41] S. Kezilebieke, M. Nurul Huda, V. Vaňo, M. Aapro, S. C. Ganguli, O. J. Silveira, S. Głodzik, A. S. Foster, T. Ojanen, and P. Liljeroth, Topological superconductivity in a designer ferromagnet-superconductor van der Waals heterostructure, Nature (London) 588, 424 (2020).

[42] A. Y. Kitaev, Unpaired majorana fermions in quantum wires, Phys. Usp. 44, 131 (2001).

[43] E. M. Stoudenmire, J. Alicea, O. A. Starykh, and M. P. A. Fisher, Interaction effects in topological superconducting wires supporting Majorana fermions, Phys. Rev. B 84, 014503 (2011).

[44] M. Tezuka and N. Kawakami, Reentrant topological transitions in a quantum wire/superconductor system with quasiperiodic lattice modulation, Phys. Rev. B 85, 140508(R) (2012).

[45] R. Thomale, S. Rachel, and P. Schmitteckert, Tunneling spectra simulation of interacting majorana wires, Phys. Rev. B 88, 161103(R) (2013).

[46] S. Gangadharaiah, B. Braunecker, P. Simon, and D. Loss, Majorana Edge States in Interacting One-Dimensional Systems, Phys. Rev. Lett. 107, 036801 (2011).

[47] A. Haim, A. Keselman, E. Berg, and Y. Oreg, Time-reversalinvariant topological superconductivity induced by repulsive interactions in quantum wires, Phys. Rev. B 89, 220504(R) (2014).

[48] M. Cheng, M. Becker, B. Bauer, and R. M. Lutchyn, Interplay Between Kondo and Majorana Interactions in Quantum Dots, Phys. Rev. X 4, 031051 (2014).

[49] A. M. Lobos, R. M. Lutchyn, and S. Das Sarma, Interplay of Disorder and Interaction in Majorana Quantum Wires, Phys. Rev. Lett. 109, 146403 (2012).

[50] S. V. Aksenov, A. O. Zlotnikov, and M. S. Shustin, Strong coulomb interactions in the problem of majorana modes in a wire of the nontrivial topological class bdi, Phys. Rev. B 101, 125431 (2020).

[51] A. Keselman and E. Berg, Gapless symmetry-protected topological phase of fermions in one dimension, Phys. Rev. B 91, 235309 (2015).
[52] L. Fidkowski, R. M. Lutchyn, C. Nayak, and M. P. A. Fisher, Majorana zero modes in one-dimensional quantum wires without long-ranged superconducting order, Phys. Rev. B 84, 195436 (2011).

[53] J. D. Sau, B. I. Halperin, K. Flensberg, and S. Das Sarma, Number conserving theory for topologically protected degeneracy in one-dimensional fermions, Phys. Rev. B 84, 144509 (2011).

[54] D. E. Parker, T. Scaffidi, and R. Vasseur, Topological luttinger liquids from decorated domain walls, Phys. Rev. B 97, 165114 (2018).

[55] N. Lang and H. P. Büchler, Topological states in a microscopic model of interacting fermions, Phys. Rev. B 92, 041118(R) (2015).

[56] T. Scaffidi, D. E. Parker, and R. Vasseur, Gapless SymmetryProtected Topological Order, Phys. Rev. X 7, 041048 (2017).

[57] R. Verresen, N. G. Jones, and F. Pollmann, Topology and Edge Modes in Quantum Critical Chains, Phys. Rev. Lett. 120, 057001 (2018).

[58] A. Keselman, E. Berg, and P. Azaria, From one-dimensional charge conserving superconductors to the gapless haldane phase, Phys. Rev. B 98, 214501 (2018).

[59] J. Ruhman, E. Berg, and E. Altman, Topological States in a One-Dimensional Fermi Gas with Attractive Interaction, Phys. Rev. Lett. 114, 100401 (2015).

[60] G. Ortiz, J. Dukelsky, E. Cobanera, C. Esebbag, and C. Beenakker, Many-Body Characterization of ParticleConserving Topological Superfluids, Phys. Rev. Lett. 113, 267002 (2014).

[61] J. Alicea, New directions in the pursuit of majorana fermions in solid state systems, Rep. Prog. Phys. 75, 076501 (2012).

[62] P. C. Hohenberg, Existence of long-range order in one and two dimensions, Phys. Rev. 158, 383 (1967).

[63] N. D. Mermin and H. Wagner, Absence of Ferromagnetism or Antiferromagnetism in one- or Two-Dimensional Isotropic Heisenberg Models, Phys. Rev. Lett. 17, 1133 (1966).

[64] F. C. Alcaraz, M. N. Barber, M. T. Batchelor, R. J. Baxter, and G. R. W. Quispel, Surface exponents of the quantum XXZ, Ashkin-Teller and Potts models, J. Phys. A: Math. Gen. 20, 6397 (1987).

[65] E. K. Sklyanin, Boundary conditions for integrable quantum systems, J. Phys. A: Math. Gen. 21, 2375 (1988).

[66] H.-J. Mikeska and A. K. Kolezhuk, One-dimensional magnetism, in Quantum Magnetism, Lecture Notes in Physics, edited by U. Schollwöck, J. Richter, D. J. J. Farnell, and R. F. Bishop (Springer Berlin Heidelberg, Berlin, Heidelberg, 2004), Vol. 645, p. 1-83, https://link.springer.com/chapter/10. 1007/BFb0119591.

[67] P. Fendley, Strong zero modes and eigenstate phase transitions in the xyz/interacting majorana chain, J. Phys. A: Math. Theor. 49, 30LT01 (2016).

[68] S.-i. Tomonaga, Remarks on Bloch's method of sound waves applied to many-fermion problems, Prog. Theor. Phys. 5, 544 (1950).

[69] J. Luttinger, An exactly soluble model of a many-fermion system, J. Math. Phys. 4, 1154 (1963)

[70] F. D. M. Haldane, 'Luttinger liquid theory' of one-dimensional quantum fluids. i. properties of the Luttinger model and their extension to the general 1d interacting spinless fermi gas, J. Phys. C: Solid State Phys. 14, 2585 (1981). 
[71] A. Weiße, G. Wellein, A. Alvermann, and H. Fehske, The kernel polynomial method, Rev. Mod. Phys. 78, 275 (2006).

[72] F. A. Wolf, J. A. Justiniano, I. P. McCulloch, and U. Schollwöck, Spectral functions and time evolution from the chebyshev recursion, Phys. Rev. B 91, 115144 (2015).

[73] J. L. Lado and O. Zilberberg, Topological spin excitations in harper-heisenberg spin chains, Phys. Rev. Res. 1, 033009 (2019).

[74] J. L. Lado and M. Sigrist, Solitonic in-gap modes in a superconductor-quantum antiferromagnet interface, Phys. Rev. Res. 2, 023347 (2020).

[75] M. Fishman, S. R. White, and E. M. Stoudenmire, The ITensor Software Library for Tensor Network Calculations, arXiv:2007.14822.

[76] ITensor Library http://itensor.org.

[77] DMRGpy Library https://github.com/joselado/dmrgpy.

[78] D. Rainis and D. Loss, Majorana qubit decoherence by quasiparticle poisoning, Phys. Rev. B 85, 174533 (2012).
[79] M. J. Schmidt, D. Rainis, and D. Loss, Decoherence of majorana qubits by noisy gates, Phys. Rev. B 86, 085414 (2012).

[80] J. C. Budich, S. Walter, and B. Trauzettel, Failure of protection of majorana based qubits against decoherence, Phys. Rev. B 85 , 121405(R) (2012).

[81] M. Fabrizio and A. O. Gogolin, Interacting one-dimensional electron gas with open boundaries, Phys. Rev. B 51, 17827 (1995).

[82] J. Von Delft and H. Schoeller, Bosonization for beginners-refermionization for experts, Ann. Phys. 7, 225 (1998).

[83] E. Casiano-Diaz, Quantum entanglement of one-dimensional spinless fermions, Master thesis, The University of Vermont, 2019, https://scholarworks.uvm.edu/graddis/1052/.

[84] M. Greiter, V. Schnells, and R. Thomale, The 1D Ising model and the topological phase of the Kitaev chain, Ann. Phys. 351, 1026 (2014). 